\title{
Modulation of single-molecule magnet behaviour via photochemical [2+2] cycloaddition
}

\author{
Long-Fei Wang, $a$ Yan-Cong Chen, $a$ Jun-Liang Liu,,$a$ Jiang Liu, $a$ Quan-Wen Li, $a$ Jun-Yu \\ Hong,,$a$ Jian-Hua Jia, ${ }_{a}$ Jesús Jover, $b$ Eliseo Ruiz* ${ }_{b}$ and Ming-Liang Tong* ${ }_{a}$
}

\begin{abstract}
The first example of photo-tunable single-ion magnet (SIM) through [2+2] cycloaddition reaction is reported. Self-assembly of $\mathrm{Dy}\left(\mathrm{NO}_{3}\right)_{3} \cdot 6 \mathrm{H}_{2} \mathrm{O}$ and 1,2-bis(4-pyridyl)ethene (bpe) ligand in $\mathrm{MeCN} / \mathrm{EtOH}$ solution generated complex $\left[\mathrm{Dy}(\mathrm{bpe})\left(\mathrm{H}_{2} \mathrm{O}\right)_{4}\left(\mathrm{NO}_{3}\right)_{2}\right] \mathrm{NO}_{3} \cdot 2 \mathrm{bpe}$ (1). Each pair of bpe ligands from two adjacent [Dy $\left.(\mathrm{bpe})\left(\mathrm{H}_{2} \mathrm{O}\right)_{4}\left(\mathrm{NO}_{3}\right)_{2}\right]^{+}$motifs successfully undergo complete [2+2] dimerization under UV light irradiation, leading to complex $\left[\mathrm{Dy} y_{2}(\mathrm{tpcb})\left(\mathrm{H}_{2} \mathrm{O}\right)_{8}\left(\mathrm{NO}_{3}\right)_{4}\right]\left(\mathrm{NO}_{3}\right)_{2} \cdot 2$ bpe.tpcb (2) (tpcb = tetrakis (4pyridyl)cyclobutane) through single crystal to single crystal transformation. Both complexes show fieldinduced slow relaxation of magnetization at low temperature. The corresponding diluted samples,

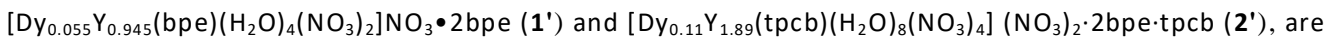
also prepared for the investigation of SIM behaviour of Dy(III) site for both lanthanides complexes excluding intermolecular interaction. Alternating current (ac) susceptibility measurements performed on the diluted samples reveal the characteristic slow relaxation behaviour in the absence of field for $\mathbf{1}^{\prime}$ with $U_{\text {eff }} / k_{\mathrm{B}}=55.2 \mathrm{~K}$ while only field-induced slow relaxation behaviour was observed for 2 ', confirming the sensitive response of the magnetic behaviour of Dy(III) ions to the variation of ligand field tuned by [2+2] reaction. The observed differences between the two complexes have been explained with the help of CASSCF/RASSI calculations.
\end{abstract}

\section{Introduction}

The solid state photochemical [2+2] cycloaddition reaction of olefinic group as a typical topochemical reaction has been studied extensively. ${ }^{1,2}$ According to Schmidt's criterion, through the rational topochemical alignment of ethylenic double bonds in ligand, the [2+2] cycloaddition reaction may occur under a UV light, leading to dimeric cyclobutyl product. Inspired by this unique optical activity, solid-state structural transformations by photochemical [2+2] reaction of $\mathrm{C}=\mathrm{C}$ bonds in various discrete coordination cages, coordination polymers and organic compounds have been extensively investigated in recent decade through single crystal-to-single crystal (SCSC) manner involving only simple linear olefins. ${ }^{3,4}$ On the other hand, besides the structural transformation, the variation of physical or chemical properties tuned by [2+2] cycloaddition reaction, such as conductivity ${ }^{5 a}$ and solubility, ${ }^{5 b}$ will be even more important and show potential for functionalization. However, the investigation emphasizing on bridging [2+2] cycloaddition reaction with magnetic functions has, to date, rarely been explored. ${ }^{6}$

Single-ion magnets (SIMs)
Single-molecule magnets (SMMs) that displayed slow magnetic relaxation (magnetic bistability) and magnetic hysteresis ${ }^{7,8}$ at molecule level are enticing because of potential application for high-density storage devices, spintronics, and quantum computations. ${ }^{9,10}$ In recent years, the single-ion magnets (SIMs), a class of SMMs, are becoming a subject of the extensive research due to the enhanced magnetic behaviour

${ }^{a}$ Key Laboratory of Bioinorganic and Synthetic Chemistry of Ministry of Education, State Key Laboratory of Optoelectronic Materials and Technologies, School of Chemistry \& Chemical Engineering, Sun Yat-Sen University, 510275 Guangzhou, P. R. China. E-mail: jiajh3@mail.sysu.edu.cn; tongml@mail.sysu.edu.cn

${ }^{b}$ Departament de Química Inorgànica and Institut de Recerca de Química Teòrica i Computacional, Universitat de Barcelona, Diagonal 645, E-08028 Barcelona, Spain. E-mail: eliseo.ruiz@qi.ub.es

$\dagger$ Electronic supplementary information (ESI) available: Experimental procedures, structure and crystallographic data, physical measurements, computational details. CCDC 1051853 (1), 1051854 (2), For ESI andcrystallographic data in CIF or other electronic format see DOI: $\operatorname{xxxxxxx}$ 
with high energy barriers as much as $938 \mathrm{~K} .{ }^{11,12}$ Recently, the strength and symmetry of crystal/ligand field have been proved to be a key factor to regulate the uniaxial anisotropy in $4 \mathrm{f}$ SIMs. ${ }^{13}$ Moreover, some promising reports for manipulating the coordination sphere of magnetic ions in SIMs have successfully observed the sensitivity of the magnetic properties towards perturbations of ligand field. ${ }^{14,15}$ These results not only make us a further understanding of SMMs, which is significant for designing such materials with improvement properties, but also stimulated inspiration for multi-functionalization of SMMs. ${ }^{16}$

Attracted by promising application of [2+2] reaction for photoresponsive material, herein we attempt to introduce the photochemical [2+2] reaction into SMM system. As a result, complex 1, with the formula [Dy $\left.(\mathrm{bpe})\left(\mathrm{H}_{2} \mathrm{O}\right)_{4}\left(\mathrm{NO}_{3}\right)_{2}\right] \mathrm{NO}_{3} \cdot 2 \mathrm{bpe}$, is synthesized through the reaction of bpe ligand with Dy(III) ions which are commonly used in SMMs because of the large anisotropy. In 1, the bpe ligand was directly coordinated to Dy(III) ions and adopted a parallel arrangement with a distance of $3.67 \AA$ between ethylene groups. Upon the UV irradiation for 10 hours, complex 1 still maintains its single crystallization and the coordinated bpe ligand undergo complete [2+2] transformation, leading to the irradiated product $\mathbf{2}$, $\left[\mathrm{Dy}(\mathrm{tpcb})_{0.5}\left(\mathrm{H}_{2} \mathrm{O}\right)_{4}\left(\mathrm{NO}_{3}\right)_{2}\right] \mathrm{NO}_{3} \cdot$ bpe 0.5 tpcb. Interestingly, the dimerization of coordinated bpe ligand simultaneously induced the slight but indispensable rearrangement of coordinated atoms, thus change the ligand filed imposed on Dy(III) ions, resulting in the different SIM behaviour between the before- and afterirradiated complexes.

\section{Results and discussion}

The reaction of $\mathrm{Dy}\left(\mathrm{NO}_{3}\right)_{3} \cdot 6 \mathrm{H}_{2} \mathrm{O}$ and bpe ligand in a mixture solution of $\mathrm{MeCN}$ and $\mathrm{EtOH}$ in the ratio 1:3 gave colourless rodlike crystal of complex $\mathbf{1}$ which crystallises in monoclinic space group $P 2{ }_{1} / \mathrm{c}$. The crystal of $\mathbf{1}$ is very stable in the air and can maintain its crystallinity for a long time, such behaviour may be due to a rich of hydrogen-bond contained and solventfree structure of $\mathbf{1}$. The assymetric unit of $\mathbf{1}$ contains one Dy(III) ion, three $\mathrm{NO}_{3}{ }^{-}$unit , four coordinated $\mathrm{H}_{2} \mathrm{O}$ molecules and three bpe ligands. As shown in Fig. 1a, each Dy(III) ion is ninecoordinated by eight oxygen atoms, which come from two bidentate chelate $\mathrm{NO}_{3}^{-}$unit and four water molecules, and one nitrogen donor of one terminal coordinated bpe ligand, leading to a mononuclear $\left[\mathrm{Dy}(\mathrm{bpe})\left(\mathrm{H}_{2} \mathrm{O}\right)_{4}\left(\mathrm{NO}_{3}\right)_{2}\right]^{+}$unit. Along the $\mathrm{c}$ axis of crystal cell, The cation units are hydrogen bonded with each other through an uncoordinated $\mathrm{NO}_{3}{ }^{-}$ion, resulting in supermolecular 1D chain structure with the minimum intermononuclear Dy...D Dy distance of $8.41 \AA$ (Fig. 2a).

Furthermore, a pair of bpe ligands, which coordinate to two mononuclear Dy1(III) ions respectively, are related by crystallographic center of inversion and anchored through the complementary $\mathrm{N} \cdots \mathrm{H}^{-} \mathrm{O}$ hydrogen bond $(2.79 \AA)$ between the uncoordinated $\mathrm{N} 2$ atom of bpe ligand and $\mathrm{O} 3 \mathrm{~W}$ atom of $\mathrm{H}_{2} \mathrm{O}$ molecule (Fig. 2b). As a result, the two mononuclear Dy(III) unit embrace with each other through the bpe arms. The olefinic bonds of bpe ligands (N1 to N2) is parallel alignment with each other and the distance between the parallel ethylene groups of be ligands is $3.67 \AA$, indicating the potential for $[2+2]$ photochemical cycloaddition reaction $(<4.2 \AA)$. On the other hand, each pair of coordinated bpe ligands are separated with a guest bpe (N6 to N6A) molecule, leading to a slipstacked arrangement of bpe ligands along the a direction with AAB model (Fig. 2b). Although the center to center distance of $\mathrm{C}=\mathrm{C}$ bonds between free and coordinated bpe ligands is $3.83 \AA$, which also comply with Schmidt's topochemical criterion, it is most likely that inter-mononuclear photodimerization reaction would proceed as its distance is shorter. ${ }^{17}$
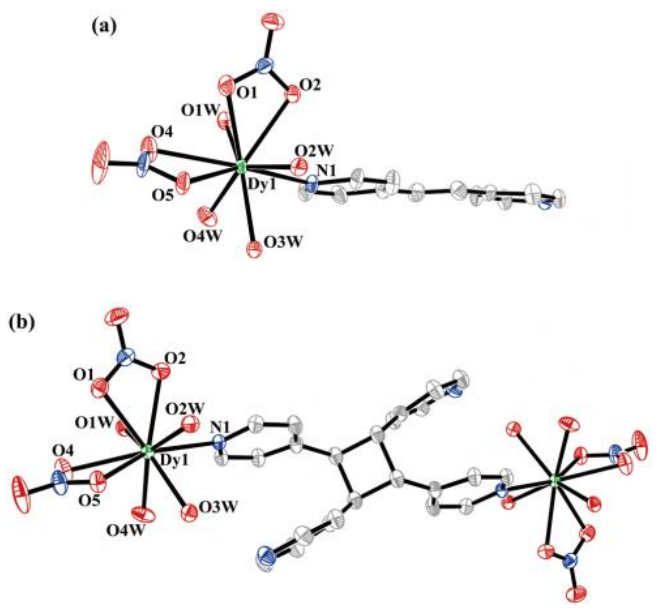

Fig. 1 The coordinated environments of Dy(III) in mononuclear [Dy] and dinuclear [Dy 2 unit of complex $\mathbf{1}(\mathrm{a})$ and $\mathbf{2}$ (b) respectively.

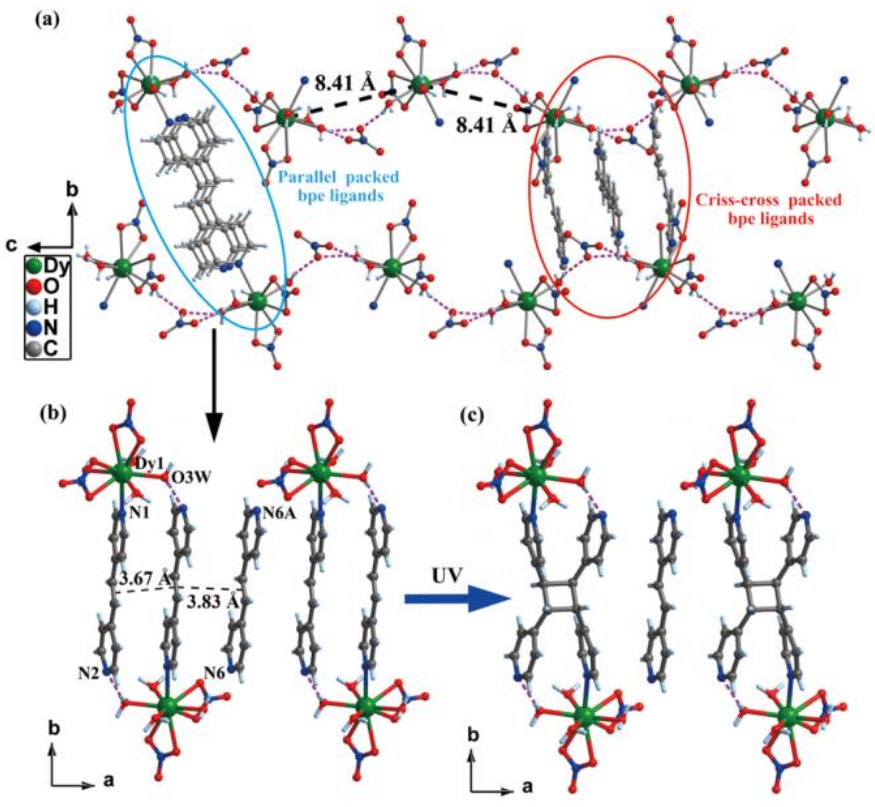

Fig. 2 (a) The hydrogen bonded 1D chain constructed by mononuclear [Dy $($ bpe $\left.)\left(\mathrm{H}_{2} \mathrm{O}\right)_{4}\left(\mathrm{NO}_{3}\right)_{2}\right]^{+}$units and guest $\mathrm{NO}_{3}{ }^{-}$ions, the parallel and criss-cross packed bpe ligands are marked by blue and red circles respectively. For clarity, the other repetitive parallel and criss-cross packed bpe ligands are omited; ( $b$ and $c$ ) The [2+2] cycloaddition reaction in parallel packed bpe ligands after UV light exposure of $\mathbf{1}$. The hydrogen bonds are displayed as purple dashed lines. 
Besides the parallel arrangement of bpe ligands, interestingly, another contrary packing model of $\mathrm{C}=\mathrm{C}$ bonds were also observed. Three guest bpe molecules are closely packed with each other through the hydrogen bond interaction between the $\mathrm{N}$ atom of pyridyl group and $\mathrm{O}$ atoms of $\mathrm{H}_{2} \mathrm{O}$ molecules and obvious $\pi \cdots \pi$ interaction (Figs. $2 \mathrm{a}$ and S3a). The middle bpe ligand locating on a symmetric center adopts a criss-cross alignment of $\mathrm{C}=\mathrm{C}$ bonds with the other two on both sides. The distance between adjacent centers of $\mathrm{C}=\mathrm{C}$ bonds is $3.49 \AA$.

Because of the close proximity of the olefinic in $\mathbf{1}$ raising the prospect of a solid state photochemical [2+2] cycloaddition reaction, the single crystal of $\mathbf{1}$ was subjected to UV irradiation at room temperature by an ultra-high pressure mercury lamp $(P=300 \mathrm{~W})$ for 10 hours, resulting in the irradiated product 2. Single-crystal $\mathrm{X}$-ray analysis revealed that complex 2 crystallises in the same space group $\left(P 2_{1} / \mathrm{c}\right)$ with 1 (Table S1). However, some slight but visible variations of unit cell parameters of $\mathbf{2}$ were observed compared to $\mathbf{1}$, eg. the $\beta$ angle of $2\left(93.7^{\circ}\right)$ is larger than that of $\mathbf{1}\left(90.5^{\circ}\right)$. A following refinement of the structure of $\mathbf{2}$ revealed the occurene of a quantitative photo dimerization reaction accompanied by SCSC transformation. In complex $\mathbf{2}$, as our expected, the coordinated bpe ligands took place $100 \%$ formation of rctt-tpcb while the guest bpe ligand (N6 to N6A) with a relatively longer contact between $\mathrm{C}=\mathrm{C}$ bonds is unreactive (Fig. 2b). The formation of new covalent bonds results in conversion of the mononuclear Dy(III) unit to the dinuclear [Dy 2$]$ unit (Fig. 1b). Compared the structure of dinuclear $\left[\mathrm{Dy}_{2}\right]$ ion of $\mathbf{2}$ to mononuclear [Dy] ion of 1, although the coordinated atoms of the Dy (III) ion didn't changed, the $\pi \cdots \pi$ stacked pyridyl group of bpe ligands diverged with each other in 2 , such behaviour indirectly elongate the Dy $1 \cdots \mathrm{O} 3 \mathrm{~W}$ distance from $2.39 \AA$ to $2.44 \AA$ through the hydrogen bond interaction between uncoordinated N2 atom and $\mathrm{O} 1 \mathrm{~W}$ atom. In additional, the other atoms around Dy(III) ion also proceed slight replacement, which may be as a result of the simulation by the formation of cyclobutane derivative, leading to considerable variation of bond lengths and bond angles of coordinated atoms around Dy(III) ion (Table S2). Simultaneously, a SHAPE analysis of Dy(III) ion for the before- and after- irradiated complexes suggested a coordination geometry change between a spherical capped square antiprism $\left(\mathrm{C}_{4 \mathrm{v}}\right)$ and a spherical tricapped trigonal prism $\left(\mathrm{D}_{3 \mathrm{~h}}\right.$ ) for 1 and $\mathbf{2}$ (Table S3).

For the criss-cross packed bpe ligands, some reports have demonstrated the possibility to form $r t c t$-tpcb, a stereoisomer of rctt-tpcb, by [2+2] reaction, ${ }^{18}$ instead, the structural refinement indicated the formation of rctt-tpcb, which may owning to the pedal motion of criss-cross aligned $\mathrm{C}=\mathrm{C}$ groups of bpe ligands. ${ }^{19}$ Because of the odd number and center symmetric packed model of bpe ligands, the middle bpe ligand took place $[2+2]$ dimerization with one of the bpe ligands on both sides, finally giving one rctt-tpcb and one bpe molecule at last (Fig. $\mathrm{S} 3 \mathrm{~b}, \mathrm{c})$.

As described above, due to the photo-induced cycloaddition reaction, the coordination sphere of Dy(III) was varied between the before- and after- irradiated products. Such behaviour inspired us to investigate the response of SMM property of Dy(III) ion when considering the possible change of local ligand/crystal field. The result are stated below.

Temperature-dependent magnetic susceptibilities of both $\mathbf{1}$ and 2 were performed in a direct-current (dc) field of 1000 Oe. At room temperature, the $\chi_{M} T$ values were 13.9 and $14.2 \mathrm{~cm}^{3} \mathrm{~K}$ $\mathrm{mol}^{-1}$ for complex $\mathbf{1}$ and $\mathbf{2}$ respectively, which is agreeable with the expected value of one Dy(III) ion $\left(14.2 \mathrm{~cm}^{3} \mathrm{~K} \mathrm{~mol}^{-1}\right.$ for the ${ }^{6} H_{15 / 2}$ state). ${ }^{20}$ Upon cooling, the $\chi_{M} T$ value of $\mathbf{1}$ and $\mathbf{2}$ gradually decreases to 12.0 and $11.5 \mathrm{~cm}^{3} \mathrm{~K} \mathrm{~mol}^{-1}$ respectively. Taking account of the unquenched orbital moment accompanied with a ligand field of Dy(III) ion, the decrease of $\chi_{M} T$ value of 1 and 2 is mostly attributed to the progressive depopulation of Stark sublevels of $4 f \mathrm{Dy}$ (III) ion. The magnetization (Fig. 3) increase rapidly with field strength up to about $0.9 \mathrm{~T}$ and then gradually increased slowly, reaching the "saturation" of $6.05 N \beta$ for $\mathbf{1}$ and $5.93 N \beta$ for 2 , indicating the existing magnetic anisotropy.

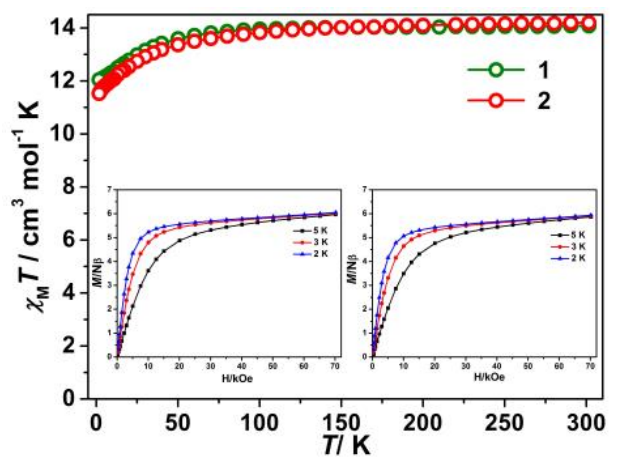

Fig. 3 Temperature dependence of $\chi_{M} T$ products at 1000 Oe for 1 (green) and $\mathbf{2}$ (red). Inset: plots of $\mathrm{M}-\mathrm{H}$ for $\mathbf{1}$ (left) and $\mathbf{2}$ (right) at 2, 3 and $5 \mathrm{~K}$.

In order to explore the magnetic dynamics, detailed alternating-current (ac) susceptibility measurements were also performed. In the absence of a static field, the characteristic frequency dependence of out of phase signal, $\chi_{M}^{\prime \prime}$, was observed for 1 and 2 below around $10 \mathrm{~K}$. For 1, although an inconspicuous shoulder was found at about 5-6 $\mathrm{K}$ in both $\chi_{M}{ }^{\prime}$ and $\chi_{M}{ }^{\prime \prime}$ vs. $T$ curves when the frequency was $1488 \mathrm{~Hz}$ (Fig. $\mathrm{S} 4 \mathrm{a}$ ), at lower frequency, no maxima in out of phase signals were observed above $1.8 \mathrm{~K}$ as well as 2 (Fig. S4b), suggesting the existing fast quantum tunneling of magnetization (QTM) which accelerate the relaxation of spin-reversal process in both complexes. To suppress the QTM effect, a series of external dc fields in the range of $0.2-2.5 \mathrm{kOe}$ were applied at $2 \mathrm{~K}$. In the plot of $\chi_{M}^{\prime \prime}$ vs. $v$ at $2 \mathrm{~K}$ for 1 (Fig. S5a), the peak with a maximum at $900 \mathrm{~Hz}$ at 0 Oe disappears and a set of peaks at lower frequency region were observed at dc fields above 400 Oe. For complex 2, such filed-induced slow relaxation behaviour (Fig. S5b) was similar with 1, suggesting the suppression of QTM by application of dc field. The $1000 \mathrm{Oe} \mathrm{dc}$ field was chosen as the optimum field which result in a maximum of the relaxation time for both complexes.

On the other hand, it is noteworthy that although the slow relaxation behaviours for $\mathbf{1}$ and $\mathbf{2}$ were observed due to application of external dc field in Fig. S5, as the increase of dc 
field, the $\chi_{M}{ }^{\prime \prime}$ signal increased again at lower frequency regime, suggesting another slower relaxation process. The Argand plots (Fig. S6) reporting $\chi_{M}{ }^{\prime \prime}$ vs. $\chi_{M}{ }^{\prime}$ further indicated the dualrelaxation process for both complexes above 1200 Oe dc field, where the formation of a second semicircle at low frequency was observed. However, in the structure of $\mathbf{1}$ and 2, all Dy(III) ions were equivalent, such very slow relaxation process was likely attributed to intermolecular interaction which lead to a collective spin relaxation. ${ }^{[21]}$ For the magnetic separation of Dy(III) ions, the magnetic ion sites of $\mathbf{1}$ was diluted with diamagnetic $\mathrm{Y}(\mathrm{III})$ ions with Dy: Y molars ratios of 1:17, giving the corresponding diluted sample $\mathbf{1}$ ' for $\mathbf{1}$. The diluted sample 2 ' for $\mathbf{2}$ was obtained through the UV irradiation of 1'. The structures of $\mathbf{1}^{\prime}$ and $\mathbf{2}^{\prime}$ were isomer to their undiluted $\mathbf{1}$ and $\mathbf{2}$, respectively, as confirmed by PXRD measurements (Fig. S1). The frequency dependent ac measurements at various dc field for 1' and 2' were also performed (Fig. S7). As our expect, upon dilution, the slower processes were no longer visible within the frequency range probed (1-1488 Hz). Additionally, the faster relaxation processes observed in $\mathbf{1}$ and $\mathbf{2}$ was shift to lower frequency regime for $\mathbf{1}^{\prime}$ and 2 '. The peaks of $\chi_{M}{ }^{\prime \prime}$ signal which correspond to a maximum of relaxation time for $\mathbf{1}^{\prime}$ and 2' was located at 2 and $0.2 \mathrm{~Hz}$ respectively at their optimum fields (300 Oe for $\mathbf{1}^{\prime}$ and 400 Oe for $\mathbf{2}^{\prime}$ ), lower than the corresponding frequency for $\mathbf{1}(7 \mathrm{~Hz})$ and $\mathbf{2}(3 \mathrm{~Hz})$, suggesting the slowing down of the faster relaxation processes in diluted samples.

The field induced SIM behaviour for 1 was measured under a $1 \mathrm{kOe}$ dc field (Fig. 4a), a set of peaks in $\chi_{M}{ }^{\prime \prime}$ signal was observed below $6 \mathrm{~K}$. The magnetization-relaxation time, $\tau$, was extracted by fitting the Cole-Cole plot data in the temperature range of 1.8-6.0 K with a generalized Debye model for a singlerelaxation process (Fig. S8a and S9a). The distribution coefficient values $(\alpha)$ were 0.049-0.275 (Table S4). The high temperature data fitted by Arrhenius law gave $U_{\text {eff }} / k_{\mathrm{B}}=39.4 \mathrm{~K}$, $\tau_{0}=1.30 \times 10^{-7} \mathrm{~s}$ (Fig. S10). For the diluted sample 1', the peaks in $\chi_{M}{ }^{\prime \prime}$ signals were observed at zero $\mathrm{dc}$ field, characteristic of a SIM behaviour (Fig. 5a and S11). At lower temperature, the increase of $\chi_{M}$ " signals was attributed to quantum tunneling process. The data of Cole-Cole plots (Fig. $\mathrm{S} 12)$, including relaxation time $(\tau)$ and distribution value $(\alpha)$, was summarized in Table S5. Following the Arrhenius law, the energy barrier $\left(U_{\text {eff }} / k_{\mathrm{B}}\right)$ was obtained as $55.2 \mathrm{~K}$ with $\tau_{0}=9.81 \times$ $10^{-9} \mathrm{~s}$ (Fig. 6a). The ac measurement for $\mathbf{1}^{\prime}$ was further performed at an optimum field (300 Oe) (Fig. 5b and S13a), obviously, the QTM effect was suppressed and more frequency-dependent peaks of $\chi_{M}{ }^{\prime \prime}$ signals were observed compared to the $\chi_{M}{ }^{\prime \prime}$ vs. $T$ plot for 1 ' at zero field. The $U_{\text {eff }} / k_{\mathrm{B}}$ and $\tau_{0}$ was obtained as $56.7 \mathrm{~K}$ and $8.92 \times 10^{-9} \mathrm{~s}$, respectively, by Arrhenius fitting (Fig. 6a).

For the irradiated product 2 , the $\chi_{M}$ " signals were measured at 1000 Oe field (Fig. 4b) as same as $\mathbf{1}$, at the frequency of $1488 \mathrm{~Hz}$, the peak of $\chi_{M}{ }^{\prime \prime}$ signals was observed at about $5 \mathrm{~K}$, a little lower than peak temperature $(5.8 \mathrm{~K})$ for $\mathbf{1}$ at the same frequency. The resulting data, including $\tau$ and $\alpha$ by fitting the Cole-Cole plot (Fig. S9b) was shown in Table S4. Fitting the five highest temperature point of $\mathbf{2}$ to Arrhenius law yields values of $U_{\mathrm{eff}} / k_{\mathrm{B}}=45.5 \mathrm{~K}, \tau_{0}=1.84 \times 10^{-8} \mathrm{~s}$ (Fig. S10). For diluted 2', although a shoulder peak was observed at the highfrequency limit $(1488 \mathrm{~Hz})$ of our magnetometer in the absence of static field (Fig. 5c), however, the $\chi_{M}$ " signals increase fast below $3.5 \mathrm{~K}$ due to quantum tunneling effects at zero dc field. By applying a 400 Oe external dc field, a set of peaks in $\chi_{M}{ }^{\prime \prime}$ signal at the frequency range of $1-1488 \mathrm{~Hz}$ was observed (Fig. $5 d$ ), indicating the effective suppression of QTM in 2'. At the lower frequency range, $0.1-1 \mathrm{~Hz}$, the peaks of $\chi_{M}$ " signals at 1.8 and $2.2 \mathrm{~K}$ were further found at 0.2 and $0.5 \mathrm{~Hz}$ respectively in $\chi_{M}$ " vs. $v$ plot shown in Fig. S13b, suggesting one order larger of relaxation time $(\tau)$ for $\mathbf{2}$ ' than $\mathbf{2}$ at low temperature. The relaxation time $(\tau)$ and distribution coefficient values $(\alpha)$ obtained from Cole-Cole plots (Fig. S14b) were shown in Table S7. The energy barrier was obtained by fitting high temperature data of $\ln (\tau)$ with Arrhenius law, as $U_{\text {eff }} / k_{\mathrm{B}}=47.93 \mathrm{~K}, \tau_{0}=1.22$ $\times 10^{-9} \mathrm{~s}$ (Fig. 6a).
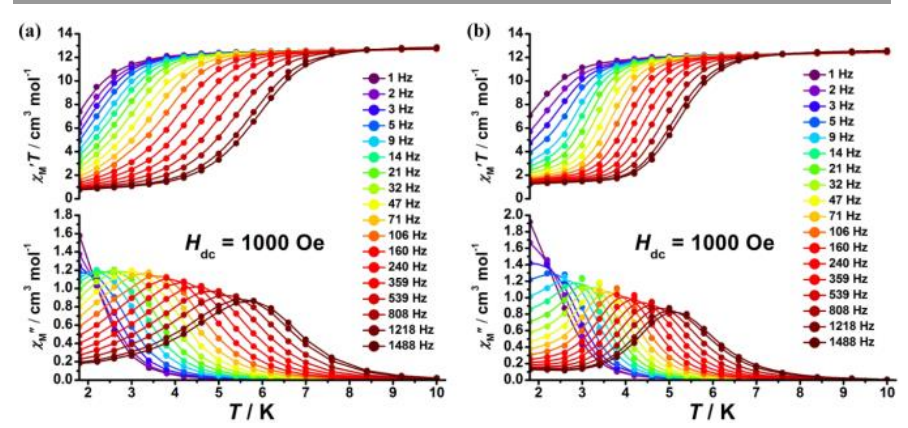

Fig. 4 The temperature dependence of ac susceptibility for $\mathbf{1}$ (a) and $\mathbf{2}$ (b) under the 1000 Oe dc field $(1-1488 \mathrm{~Hz}, 1.8-10 \mathrm{~K})$.
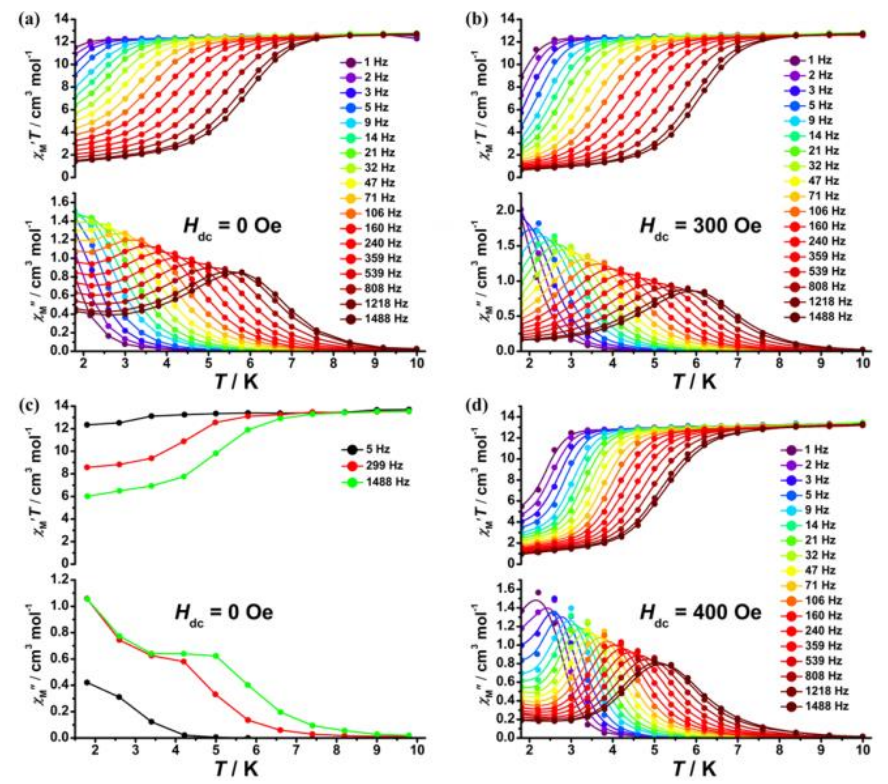

Fig. 5 The temperature dependence of ac susceptibility for diluted samples $\mathbf{1}^{\prime}(\mathrm{a}, \mathrm{b})$ and $\mathbf{2}^{\prime}(\mathrm{c}, \mathrm{d})$ under zero dc field and their optimal field, 300 Oe for $\mathbf{1}^{\prime}$ and 400 Oe for $\mathbf{2}^{\prime}$. 
Obviously, upon dilution, the thermally activated relaxation barriers for 1' and 2' were larger than their undiluted isomorphs due to the removal of intermolecular interaction. Considering the magnetic ion-ion separation in the diluted samples 1' and 2', the slow relaxation processes were more likely attributed to single ion behaviour. Thus, the analyses of the variation of the magnetic behaviour through [2+2] reaction were only performed between $\mathbf{1}^{\prime}$ and $\mathbf{2}^{\prime}$. For both complexes, the most obvious variation was the different slow relaxation characteristics at zero field, for $\mathbf{1}$, the clear relaxation process in $\chi_{M}{ }^{\prime \prime}$ signal which characteristic of a SIM behaviour with an energy barrier of $55.2 \mathrm{~K}$ at zero field was observed at the frequency range of $1-1488 \mathrm{~Hz}$ while the fast increase of $\chi_{M}{ }^{\prime \prime}$ value at low temperature due to QTM dissembled the thermally activated relaxation in 2 '. By applying the optimum field for both complexes, the resulting energy barriers for $\mathbf{1}^{\prime}(56.7 \mathrm{~K})$ and $\mathbf{2}^{\prime}(47.9 \mathrm{~K})$ further confirm the change of thermally activated relaxation tuned by $[2+2]$ reaction.
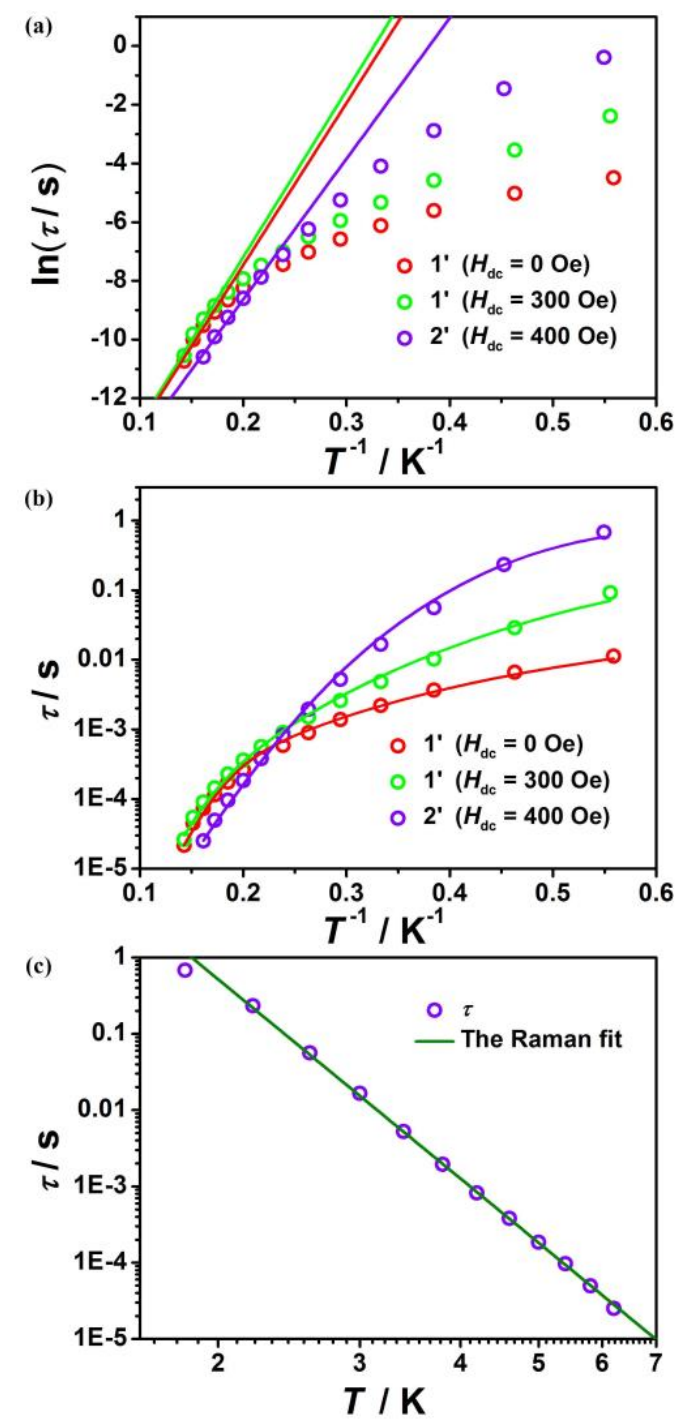

Fig. 6 (a) The $\ln (\tau)$ vs $T^{-1}$ plot for $\mathbf{1}^{\prime}$ under zero (red) and 300 (green) Oe field; for 2' (blue) under 400 Oe dc field, The solid lines are best fits with
Arrhenius law; (b) a complete fitting of the magnetisation relaxation times of $\mathbf{1}^{\prime}$ and $\mathbf{2}^{\prime}$ at different fields; (c) the plot of the relaxation time $\tau$ vs. $T$ for $\mathbf{2}^{\prime}$ on a log-log scale, The solid green lines correspond to the apparent Raman fitting.

Moreover, as shown in Fig. 6a, at lower temperature, the relaxation times, $\tau$, of both complexes were deviated from Arrhenius plots. Such behaviour was attributed to the ruling out of Orbach process as dominating relaxation mechanism at the low temperature regime. Indeed, we found the relaxation time, $\tau$, for $2^{\prime}$ was well fitted with an experimental $T^{n}$ law with $n=$ 8.68 (Fig. 6c). For a pure two phonon Raman process, it has been theoretically predicted $n=9$ for Kramer ions. ${ }^{[22,23]}$ Herein, the obtained $n$ value for $\mathbf{2}^{\prime}$ was in good agreement with the theoretical value, suggesting the dominated two phonon Raman process for $2^{\prime}$ at the measured temperature of $1.8-6.2 \mathrm{~K}$. However, the linearity of $\tau$ vs. $T$ plot on a log-log scale for $\mathbf{1}^{\prime}$ at zero and 300 Oe field were all neither as well as 2' (Fig S15). In view of the admixture of four relaxation processes (QTM, Orbach, direct and Raman) in both complexes, a complete fitting which took account $\mathrm{t}$ simultaneously both temperature and field dependent relaxation time data was performed with the reported equation (1). ${ }^{[24]}$

$\tau-1=A H_{2} T+B_{1} /\left(1+B_{2} H_{2}\right)+C T_{n}+\tau 0-1 \exp \left(-U_{\text {eff }} / k \mathrm{~B} T\right)$

In (1), the four terms, $A H^{2} T, B_{1} /\left(1+B_{2} H^{2}\right), C T^{\mathrm{n}}$ and $\tau_{0}^{-1} \exp (-$ $\left.U_{\text {eff }} / k_{\mathrm{B}} T\right)$, correspond to direct, QTM, Raman and Orbach process respectively. The values of $A, B_{1}$ and $B_{2}$ parameters for 1' and 2' were extracted from their corresponding field dependent relaxation time data at $2 \mathrm{~K}$ (Fig. S16) and were locked in equation (1). With these restraints, the parameters for Raman and Orbach term were left to vary freely. The resulting parameters from the best fit (Fig. 6b) were summarized in Table S8, we noted the Raman exponent, $n$, for 2' was 8.51, which was similar with the $n$ value obtained by $T^{n}$ law, indicating the importance of Raman process. For 1', the equation (1) was used to fit the relaxation time data at both zero and 400 Oe field, the $n$ value in Raman term was 3.46 and 5.38, the increase of $n$ from $\mathbf{1}^{\prime}$ to $\mathbf{2}^{\prime}$ indicated the increased contribution of Ramen process. In contrast, the contributions of the direct processes also decrease from 1' to $\mathbf{2}^{\prime}$, as evidenced by decreasing magnitudes of $A$. The values of $U_{\text {eff }} / k_{\mathrm{B}}$ in Orbach term were 68.14 and $72.96 \mathrm{~K}$ for $\mathbf{1}^{\prime}$ at 0 and 300 Oe field respectively, larger than $48.33 \mathrm{~K}$ for $\mathbf{2}^{\prime}$, suggesting the decreased contribution from 1' to $\mathbf{2}$ ' and such trend was also in accordance with the different slow relaxation behaviour at zero field for 1' (slow relaxation observed at zero field) and 2' (field-induced slow relaxation). In additional, the extracted energy barrier from the complete fitting with equation (1) for $\mathbf{2}^{\prime}$ was slightly larger than the corresponding value obtained from Arrhenius fitting. In fact, the experimental points corresponding to 2 ' in Fig. 6a still bend down in the high-T region, which means that the Arrhenius regime should show up at still higher temperatures.

It is well known that the single-ion anisotropy of Dy(III) ion was highly sensitive to the coordination environment. In order 
to get further insight into the low-lying electronic structure and the magnetic anisotropy on the Dy(III) site, $a b$ initio calculations of the $\operatorname{CASSCF}(7,9) / \mathrm{RASSI}$ type (Molcas 8.0 code $)^{[25]}$ for three different multiplicities $(21,128$ and 98 states for sextet, quartet and doublet calculations, respectively) on the experimental structures were performed while the employed basis set has the following contractions: Dy [9s8p6d4f3g2h]; O [4s3p2d1f]; N [4s3p2d1f]; C [3s2p]; H [2s]. ${ }^{[26]}$ For the compound $\mathbf{2}$, two calculations (2a and $\mathbf{2 b}$ ) were performed replacing one of the Dy ${ }^{\mathrm{III}}$ centers by diamagnetic $\mathrm{La}^{\mathrm{III}}$ cations (using model potentials). The calculated g-factors clearly show the large anisotropic character of these systems $\left(g_{x}, g_{y}, g_{z} \mathbf{1}\right.$ : $0.17,0.33,19.09 ; \mathbf{2 a}: 0.35,0.98,18.36$ and $\mathbf{2 b}: 0.35,0.98$, 18.40). However, the shape of the the plotted beta density of the 4f Dy ${ }^{I I I}$ electrons obtained in the CASSCF step for the ground state of the two complexes is represented in Figure 7 (Dy ${ }^{\mathrm{III}}$ centers have $4 \mathrm{f}^{9}$ electron configuration and the 7 alpha electrons give an isotropic spherical electron density) ${ }^{[27]}$ showing the relatively large distortion of the beta electron density of the oblate shape ${ }^{[28]}$ despite the relatively large calculated magnetic anisotropy. The direction of the magnetic moment is slightly different between the two complexes (see Fig. 7) but is more less aligned towards the axial nitrato ligand reflecting a larger metal-ligand electron repulsion in such direction due to the negative charge of nitrato ligand. ${ }^{[27,29]}$

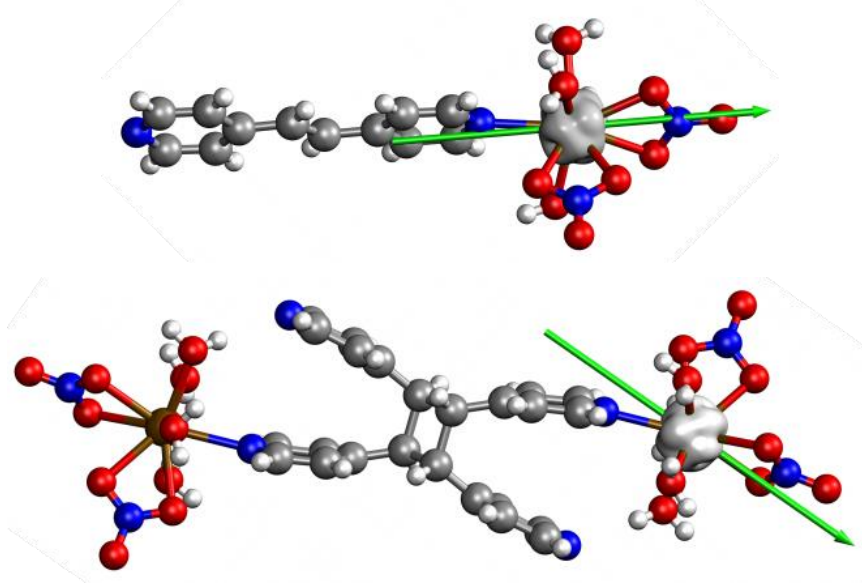

Fig 7 Beta spin density isosurface of the Dy"ll $f$ electrons for the spinfree CASSCF and the direction of the magnetic moment of the ground state is indicated as a green arrow for $\mathbf{1}$ (above) and $\mathbf{2 a}$ (below, identical result for $\mathbf{2} \mathbf{b})$.

The calculated energies of the Kramers doublets are represented in Fig. 8. The spin relaxation mechanisms indicate an effcient relaxation through the first excited state and we have a nice qualitative agreement between the first excitation energy (for $\mathbf{1}$ $78.4 \mathrm{~cm}^{-1}$ while $50.5 \mathrm{~cm}^{-1}$ for $\mathbf{2 a}$ and $\mathbf{2 b}$ ) with the experimental energy barrier values for the diluted samples $\left(50.7 \mathrm{~cm}^{-1}\right.$ and 33 $\mathrm{cm}^{-1}$ for $\mathbf{1}^{\prime}$ and $\mathbf{2}^{6}$, respectively). ${ }^{[30]}$ The analysis of the spin relaxation mechanism (see Fig. 8) reveals a larger tunneling effect at ground state level for $\mathbf{2}$ consistent with the requirement for 2' of magnetic field to present SMM behaviour while 1' is a zero-field SMM system (also it is noticed in the previously reported larger calculated $g_{x}$ and $g_{y}$ values for $\mathbf{2}$ and in the experimentally fitted $B_{1}$ value in Table S8). ${ }^{[27]}$ The calculated ground state magnetic moment is consistent with a majoritary $m_{\mathrm{J}}=15 / 2$ contribution and it is slightly larger for $\mathbf{1}$ in agrement with the experimental magnetization (see Fig. 3)
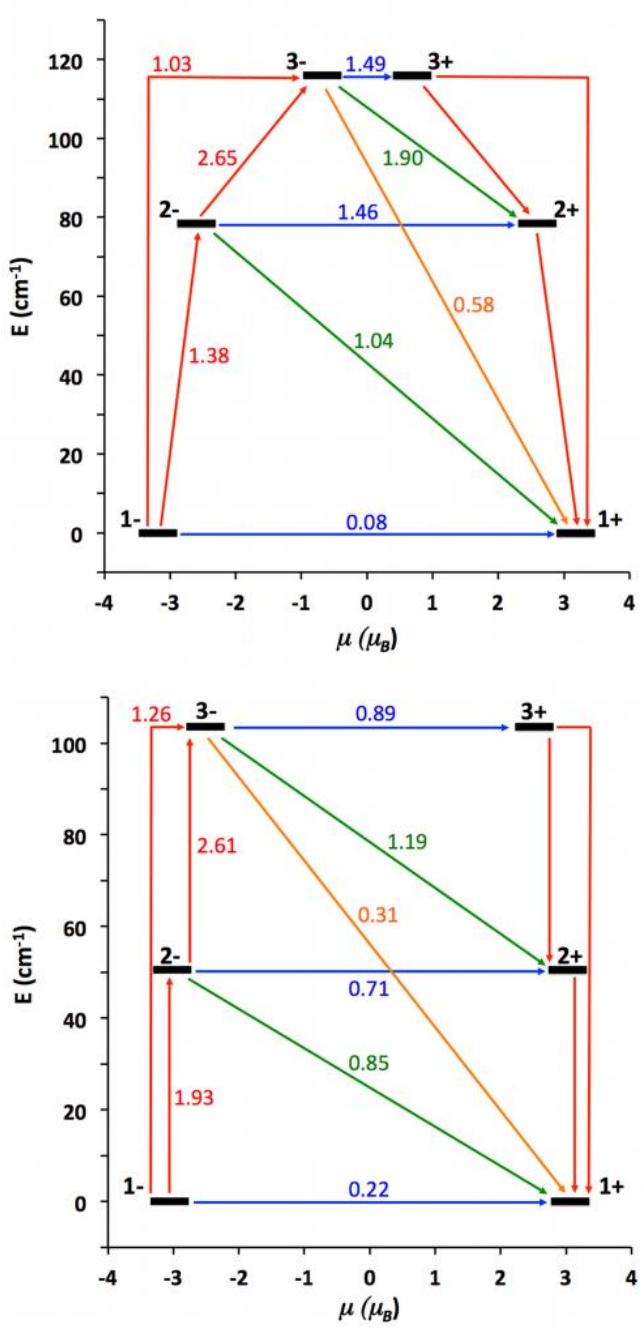

Fig. 8 Lowest three Kramers doublets and ab initio computed relaxation mechanism in $\mathbf{1}$ (above) and $\mathbf{2}$ (below). The thick black lines imply KDs as a function of their magnetic moment along the main anisotropy axis. Red lines indicates magnetization reversal mechanism the blue lines correspond to ground state QTM and thermally assisted-QTM via the first and second excited KDs, green (and orange) lines show possible Orbach relaxation processes. The values indicated close to the arrows indicate the matrix elements of the transition magnetic moments (above 0.1 is expected an effcient spin relaxation mechanism). ${ }^{[31]}$

\section{Conclusions}

\section{Acknowledgements}

This work was supported by the "973 Project" (2012CB821704 and 2014CB845602), the NSFC (Grant nos 91122032, 91422302 and 21301197), the NSF of Guangdong (S2013020013002), Program for Changjiang Scholars and Innovative Research Team in University of China, and 
the Fundamental Research Funds for the Central Universities (14lgpy10). The research reported here was also supported by the Spanish Ministerio de Economía y Competitividad (grants CTQ2011-23862-C02-01). E.R. thanks Generalitat de Catalunya for an ICREA Academia fellowship also CSUC for computer time.

1 (a) M. D. Cohen, G. M. J. Schmidt and F. I. Sonntag, J. Chem. Soc., 1964, 2000; (b) G. M. J. Schmidt, Pure Appl. Chem., 1971, 27, 647.

2 (a) I. G. Georgiev and L. R. MacGillivray, Chem. Soc. Rev., 2007, 36, 1239; (b) G. Kumar Kolea and J. J. Vittal, Chem. Soc. Rev., 2013, 42, 1755; (c) Y.-F. Han and G.-X. Jin, Acc. Chem. Res., 2014, 47, 3571.

3 (a) Q.-L. Chu, D. C. Swenson and L. R. MacGillivray, Angew. Chem. Int. Ed., 2005, 44, 3569; (b) Y.-C. Ou, D.-S. Zhi, W.-T. Liu, Z.-P. Ni, and M.-L. Tong, Chem. Eur. J., 2012, 18, 7357; (c) Y.-C. Ou, W.-T. Liu, J.-Y. Li, G.-G. Zhang, J. Wang and M.-L. Tong, Chem. Commun., 2011, 47, 9384; (d) R. Medishetty, T. T. S. Yap, L. L. Koh and J. J. Vittal, Chem. Commun., 2013, 49, 9567; (e) Y.-F. Han, G.X. Jin and F. E. Hahn, J. Am. Chem. Soc., 2013, 135, 9263; (f) D. Samanta and P. S. Mukherjee, J. Am. Chem. Soc., 2014, 136, 17006; (g) Y.-F. Han, G.-X. Jin, C. G. Daniliuc and F. E. Hahn, Angew. Chem. Int. Ed., 2015, 54, 4958.

4 (a) D. Liu, Z.-G. Ren, H.-X. Li, J.-P. Lang, N.-Y. Li and B. F. Abrahams, Angew. Chem. Int. Ed., 2010, 49, 4767; (b) I.-H. Park, A. Chanthapally, Z.-J. Zhang, S. S. Lee, M. J. Zaworotko and J. J. Vittal, Angew. Chem. Int. Ed., 2014, 53, 414; (c) I.-H. Park, R. Medishetty, J.-Y. Kim, S. S. Lee and J. J. Vittal, Angew. Chem. Int. Ed., 2014, 53, 5591; (d) I.-H. Park, A. Chanthapally, H.-H. Lee, H. S. Quah, S. S. Lee and J. J. Vittal, Chem. Commun., 2014, 50, 3665.

5 (a) K. M. Hutchins, T. P. Rupasinghe, L. R. Ditzler, D. C. Swenson, J. R. G. Sander, J. Baltrusaitis, A. V. Tivanski and L. R. MacGillivray, J. Am. Chem. Soc., 2014, 136, 6778; (b) S. Ghorai, J. C. Sumrak, K. M. Hutchins, D.-K. Bučar, A. V. Tivanski and L. R. MacGillivray, Chem. Sci., 2013, 4, 4304; (c) R. Medishetty, A. Husain, Z.-Z. Bai, T. Runčevski, R. E. Dinnebier, P. Naumov, and J. J. Vittal, Angew. Chem. Int. Ed., 2014, 53, 5907; (d) H. Sato, R. Matsuda, M. H. Mira and S. Kitagawa, Chem. Commun., 2012, 48, 7919.

6 (a) X.-Y. Wang, Z.-M. Wang and S. Gao, Chem. Commun., 2007, 11, 1127; (b) A. Chanthapally, G. K. Kole, K. Qian, G. K. Tan, S. Gao and J. J. Vittal, Chem. Eur. J., 2012, 18, 7869.

7 (a) R. Sessoli, H. L. Tsai, A. R. Schake, S. Wang, J. B. Vincent, K. Folting, D. Gatteschi, G. Christou and D. N. Hendrickson, J. Am. Chem. Soc., 1993, 115, 1804; (b) R. Sessoli, D. Gatteschi, A. Caneschi and M. A. Novak, Nature, 1993, 365, 141; (c) M. N. Leuenberger and D. Loss, Nature, 2001, 410, 789.

8 D. Gatteschi, R. Sessoli and J. Villain, Molecular Nanomagnets, Oxford University Press, New York, 2006.

9 (a) R. Vincent1, S. Klyatskaya, M. Ruben, W. Wernsdorfer and F. Balestro1, Nature, 2012, 488, 357; (b) M. Urdampilleta, S. Klyatskaya, J.-P. Cleuziou, M. Ruben and W.Wernsdorfer, Nat. Mater., 2011, 10, 502.

10 (a) M. N. Leuenberger and D. Loss, Nature, 2001, 410, 789; (b) A. Ardavan, O. Rival, J. J. L. Morton, S. J. Blundell, A. M. Tyryshkin, G. A. Timco and R. E. P. Winpenny, Phys. Rev. Lett., 2007, 98, 057201-1; (c) P. C. E. Stamp and A. Gaita-Ariño, J. Mater. Chem., 2009, 19, 1718; (e) S.-Y. Yang, X.-L. Deng, R.-F. Jin, P. Naumov, M.
K. Panda, R.-B. Huang, L.-S. Zheng and B. K. Teo, J. Am. Chem. Soc., 2014, 136, 558.

11 (a) N. Ishikawa, M. Sugita, T. Ishikawa, S. Koshihara and Y. Kaizu, J. Am. Chem. Soc., 2003, 125, 8694; (b) N. Ishikawa, M. Sugita and W. Wernsdorfer, J. Am. Chem. Soc., 2005, 127, 3650; (b) J.-L. Liu, Y.-C. Chen, Y.-Z. Zheng, W.-Q. Lin, L. Ungur, W. Wernsdorfer, L. F. Chibotaru and M.-L. Tong, Chem. Sci., 2013, 4, 3310; (c) J. J. Le Roy, L. Ungur, I. Korobkov, L. F. Chibotaru, M. Murugesu, J. Am. Chem. Soc., 2014, 136, 8003; (d) R. J. Blagg, C. A. Muryn, E. J. L. McInnes, F. Tuna and R. E. P. Winpenny, Angew. Chem. Int. Ed., 2011, 50, 6530; (e) S.-D. Jiang, B.-W. Wang, G. Su, Z.-M. Wang and S. Gao, Angew. Chem. Int. Ed., 2010, 49, 7448.

12 D. N. Woodruff, R. E. P. Winpenny and R. A. Layfield, Chem. Rev., 2013, 113, 5110.

13 J. D. Rinehart and J. R. Long, Chem. Sci., 2011, 2, 2078.

14 (a) M. Gonidec, E. S. Davies, J. McMaster, D. B. Amabilino and J. Veciana, J. Am. Chem. Soc., 2010, 132, 1756; (b) N. Ishikawa, Y. Mizuno, S. Takamatsu, T. Ishikawa and S. Koshihara, Inorg. Chem., 2008, 47, 10217; (c) N. Ishikawa, M. Sugita and W. Wernsdorfer, Angew. Chem. Int. Ed., 2005, 44, 2931; (d) H.-L. Wang, K. Wang, J. Tao and J.-Z. Jiang, Chem. Commun., 2012, 48, 2973.

15 (a) K. S. Pedersen, L. Ungur, M. Sigrist, A. Sundt, M. SchauMagnussen, V. Vieru, H. Mutka, S. Rols, H. Weihe, O. Waldmann, L. F. Chibotaru, J. Bendix and J. Dreiser, Chem. Sci., 2014, 5, 1650; (b) Y. Bi , Y.-N. Guo, L. Zhao, Y. Guo, S.-Y. Lin, S.-D. Jiang, J.-K. Tang, B.-W. Wang and S. Gao, Chem. Eur. J., 2011, 17, 12476.

16 (a) M. Morimoto, H. Miyasaka, M. Yamashita, and M. Irie, J. Am. Chem. Soc., 2009, 131, 9823; (b) D. Pinkowicz, M. Ren, L.-M. Zheng, S. Sato, M. Hasegawa, M. Morimoto, M. Irie, B. K. Breedlove, G. Cosquer, K. Katoh and M. Yamashita, Chem. Eur. J., 2014, 20, 12502.

17 G. K. Kole, R. Medishetty, L. L. Koh and J. J. Vittal, Chem. Commun., 2013, 49, 6298.

18 A. M. P. Peedikakkal, L. L. Koh and J. J. Vittal, Chem. Commun., 2008, 4, 441.

19 (a) A. M. P. Peedikakkal and J. J. Vittal, Chem. Eur. J., 2008, 14, 5329; (b) M. Nagarathinam and J. J. Vittal, Chem. Commun., 2008, 4, 438; (c) R. Medishetty, R. Tandiana, L. L. Koh, and J. J Vittal, Chem. Eur., J. 2014, 20, 123; (d) R. Medishetty, T. T. S. Yap, L. L. Koh and J. J. Vittal, Chem. Commun., 2013, 49, 9567.

20 (a) R. L. Carlin, Magnetochemistry, Springer-Verlag, 1986; (b) O. Kahn, Molecular Magnetism, Wiley-VCH, New York, 1993.

21 (a) K. R. Meihaus, J. D. Rinehart and J. R. Long, Inorg. Chem., 2011, 50, 8484; (b) K. R. Meihaus, J. D. Rinehart and J. R. Long, J. Am. Chem. Soc., 2010, 132, 7572; (c) P.-E. Car, M. Perfetti, M. Mannini, A. Favre, A. Caneschi and R. Sessoli, Chem. Commun., 2011, 47, 3751; d) M. Ren, S.-S. Bao, R. A. S. Ferreira, L.-M. Zheng and L. D. Carlos, Chem. Commun., 2014, 50, 7621; (e) M. Jeletic, P.-H. Lin, J. J. L. Roy, I. Korobkov, S. I. Gorelsky, and M. Murugesu, J. Am. Chem. Soc., 2011, 133, 19286.

22 (a) A. Singh and K. N. Shrivastava, Phys. Status Solidi B, 1979, 95, 273. (b) K. N. Shrivastava, Phys. Status Solidi B, 1983, 117, 437.

23 J.-L. Liu, K. Yuan, J.-D. L., L. Ungur, W. Wernsdorfer, F.-S. Guo, L. F. Chibotaru and M.-L. Tong, Inorg. Chem., 2012, 51, 8538.

24 J. M. Zadrozny, M. Atanasov, A. M. Bryan, C.-Y. Lin, B. D. Rekken, P. P. Power, F. Neese and J. R. Long, Chem. Sci., 2013, 4, 125. 
25 F. Aquilante, L. De Vico, N. Ferre, G. Ghigo, P. A. Malmqvist, P. Neogrady, T. B. Pedersen, M. Pitonak, M. Reiher, B. O. Roos, L. Serrano-Andres, M. Urban, V. Veryazov and R. Lindh, J. Comput. Chem., 2010, 31, 224.

26 (a) L. Ungur and L. F. Chibotaru, Phys. Chem. Chem. Phys., 2011, 13, 20086. (b) L. F. Chibotaru and L. Ungur, J. Chem. Phys., 2012, 137, 064112.

27 D. Aravena and E. Ruiz, Inorg. Chem., 2013, 52, 13770.

28 J. D. Rinehart and J. R. Long, Chem. Sci., 2011, 2, 2078.

29 N. F. Chilton, D. Collison, E. J. L. McInnes, R. E. P. Winpenny and A. Soncini, Nat. Commun., 2013, 4, 2551.

30 J. Ruiz, A. J. Mota, A. Rodriguez-Dieguez, S. Titos, J. M. Herrera, E. Ruiz, E. Cremades, J. P. Costes and E. Colacio, Chem. Commun., 2012, 48, 7916.

31 S. Gómez-Coca, D. Aravena, R. Morales, E. Ruiz, Coord. Chem. Rev.., 2015, 289-290, 379. 
TOC:

The first example of phototunable SIM through SCSC [2+2] cycloaddition reaction is reported, in which the remarkable variation of coordination sphere of Dy(III) ions led to the different field-induced slow relaxation magnetization relaxation.

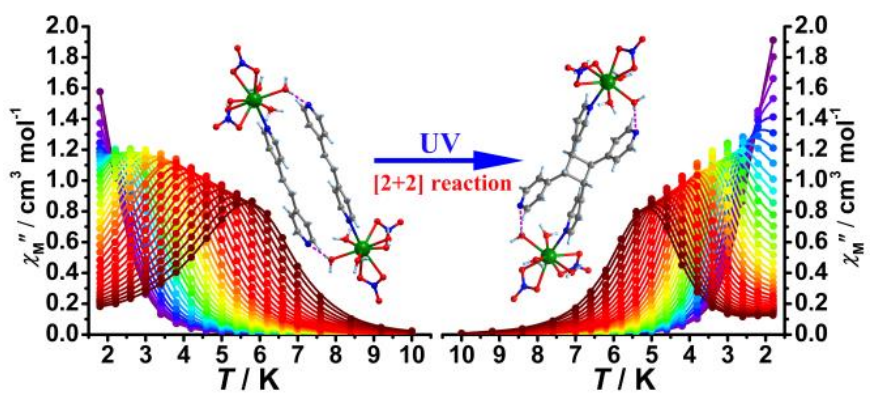

